\title{
Design of Dipole-Allowed Direct Band Gaps in Ge/Sn Core-Shell Nanowires
}

\author{
Elisabeth Pratidhina, Sunghyun Kim, and K. J. Chang*(i) \\ Department of Physics, Korea Advanced Institute of Science and Technology, Daejeon 34141, Republic of Korea
}

Supporting Information

ABSTRACT: Owing to the indirect band gap nature, Ge exhibits poor optical properties, limiting its usage for optical devices. However, since the direct band gap of $\mathrm{Ge}$ is only higher by $0.14 \mathrm{eV}$ than the indirect band gap, band gap engineering has drawn much attention to realize the direct band gap. Here, we report a strategy to design the direct band gap in $\mathrm{Ge} / \mathrm{Sn}$ core-shell nanowires (NWs), based on firstprinciples calculations. For [111]-oriented NWs, we show that the direct band gaps can be tuned by controlling the diameter and the coreto-shell ratio. We find that the intrinsic strain induced by the lattice mismatch between $\mathrm{Ge}$ and $\mathrm{Sn}$ drives an indirect-to-direct band gap transition. Even for Ge/Sn core-shell NWs with intrinsically indirect band gaps, the direct band gaps can be achieved by applying an external tensile strain lower than the critical values for pure Ge NWs and bulk

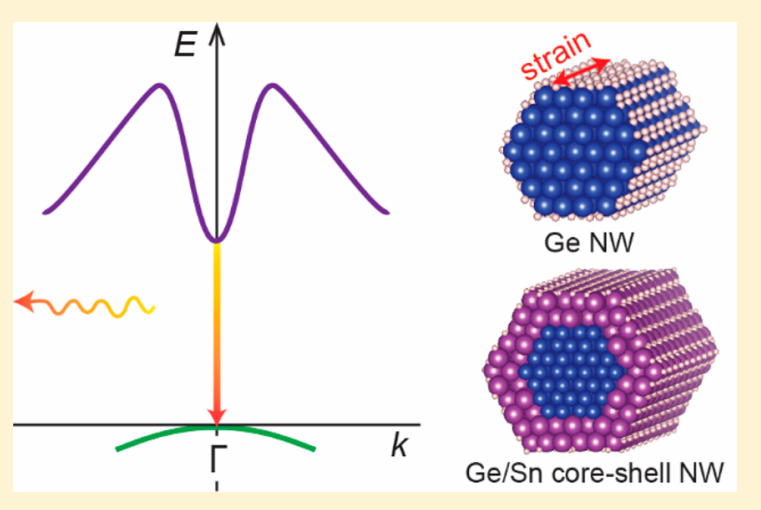
Ge. The optical transitions of the direct band gaps are all dipoleallowed, suggesting that $[111]$-oriented Ge/Sn core-shell NWs are promising for applications as light emitters.

\section{INTRODUCTION}

Semiconductor nanowires have attracted much attention because they exhibit the electrical, optical, and magnetic properties that are different from those of their bulk counterparts due to the quantum confinement effect and the large surface-to-volume ratio. ${ }^{1,2}$ In particular, germanium nanowires (Ge NWs) have been extensively studied because Ge offers the high hole mobility. ${ }^{3,4}$ A variety of potential applications using Ge NWs have been reported, such as fieldeffect transistor, ${ }^{5,6}$ photodetector, ${ }^{7}$ photoresistor, ${ }^{8}$ Li-ion battery anode, ${ }^{9}$ ultraviolet dosimetry, ${ }^{10}$ and spintronic device. ${ }^{11}$ Moreover, $\mathrm{Ge}$ is considered as a potential light source in photonic devices compatible with the current $\mathrm{Si}$ technology. ${ }^{12-14}$ However, because large-sized Ge NWs and bulk Ge have indirect band gaps, ${ }^{15,16}$ electron-hole pair recombination can only take place with phonon assistance.

Despite the indirect band gap nature, Ge has the direct band gap that only differs by $0.14 \mathrm{eV}$ from the indirect band gap, while the direct band gap is higher by about $2.3 \mathrm{eV}$ than the indirect band gap in $\mathrm{Si}^{14}$ The small difference between the indirect and direct band gaps promotes extensive studies on the band structure engineering of $\mathrm{Ge}$, such as through nanostructuring, $^{17,18}$ strain introduction, ${ }^{19-23}$ and alloying with other group-IV elements. ${ }^{24-29}$ Zone-folding and quantum confinement effects can induce direct band gaps for Ge NWs without strain, which are oriented along the [100] and [110] directions. $^{15,16}$ However, their optical transitions at the threshold energy are not improved due to the indirect gap nature of bulk Ge. Previous theoretical studies reported that strain can reduce the difference between the indirect and the direct band gaps. ${ }^{19-23}$ In particular, biaxial tensile strain works more effectively than uniaxial strain in deriving the indirect-todirect gap transition. In the case of uniaxial strain, the critical strain was shown to be generally lower along the [111] direction, while its theoretically predicted values range from $3.7 \%$ to $5.7 \% .^{20-23}$ Experimentally, the effect of biaxial tensile strain was mostly studied for bulk Ge, with the induced strains up to $2.33 \% .^{30-36}$ On the other hand, $5.7 \%$ uniaxial tensile strain along the [100] axis was achieved in suspended Ge wires on a Si substrate. ${ }^{37}$ For [111]-oriented Ge NWs, relatively low uniaxial strains were induced, such as $1.48 \%$ uniaxial strain by using silicon nitride stressor layers and $2.5 \%$ uniaxial strain by using a micromechanical 3-point stress module. ${ }^{38,39}$ Recently, it has been reported that $\mathrm{GeSn}$ alloy with the $\mathrm{Sn}$ content of about $12.6 \%$ has a direct band gap of $0.55 \mathrm{eV}$, permitting the demonstration of lasing. ${ }^{29}$ Although the direct band gap can be achieved by increasing the Sn concentration in GeSn alloys, the band gap is much reduced because $\mathrm{Sn}$ has zero gap. On the other hand, Ge NWs take advantage of the quantum confinement effect which enhances the band gap size. Since introducing Sn layers in the shell part of Ge NWs can induce intrinsic tensile strain due to the large lattice mismatch between Sn and $\mathrm{Ge}\left(a_{\mathrm{Ge}}=5.65 \AA, a_{\mathrm{Sn}}=6.48 \AA\right),{ }^{40}$ it may overcome the difficulty of accessing large strains during a microfabrication process. Therefore, based on $\mathrm{Ge} / \mathrm{Sn}$ core-shell structures, one

Received: August 31, 2016

Revised: November 17, 2016

Published: November 18, 2016 
may achieve tunable direct band gaps around $1 \mathrm{eV}$ suitable for optical applications.

In this work, we perform first-principles density functional calculations to investigate the electronic and optical properties of $\mathrm{Ge} / \mathrm{Sn}$ core-shell nanowires oriented along the [111] direction. We propose that the band gaps can be tuned by controlling the wire diameter and the ratio of the Ge core and the $\mathrm{Sn}$ shell. We find that the intrinsic strain induced by the lattice mismatch between Ge and Sn drives an indirect-to-direct band gap transition. For $\mathrm{Ge} / \mathrm{Sn}$ core-shell $\mathrm{NWs}$ with intrinsically indirect band gaps, we show that an external tensile strain along the [111] direction can trigger an indirectto-direct band gap transition; however, the critical strain is significantly reduced, as compared to pure Ge NWs with the same core sizes. We examine the matrix element of direct optical transition and find that all the direct band gaps are dipole-allowed, suggesting that our proposed NWs can serve as an efficient light emitter.

\section{CALCULATION METHOD}

Our first-principles calculations were performed by using the local density approximation (LDA) $)^{41,42}$ for the exchangecorrelation potential within the density functional theory framework and the projector augmented wave (PAW) pseudopotentials, $^{43,44}$ as implemented in the VASP code. ${ }^{45,46}$ The wave functions were expanded in plane waves up to a kinetic energy cutoff of $250 \mathrm{eV}$, and a set of $k$-points along the wire axis was generated by using the $1 \times 1 \times 8$ MonkhorstPack mesh for Brillouin zone (BZ) integration. ${ }^{47}$ Nanowire structures were modeled by including a vacuum region in the supercell geometry, in which adjacent wires were separated by about $10 \AA$, ensuring for prohibiting wire-wire interactions, and the wire surfaces were passivated with hydrogen. Both the lattice parameters and the ionic coordinates were fully relaxed until the residual forces were less than $0.04 \mathrm{eV} / \AA$. For optimized geometries, the nature of the band gaps (direct vs indirect) was examined by using a dense $k$-point mesh. Since the band gaps are underestimated by the LDA, we additionally carried out the hybrid functional calculations to confirm the band gap nature for selected nanowires, in which the HSE06 functional of Heyd, Scuseria, and Ernzerhof ${ }^{48,49}$ was used, with the screening parameter of $\omega=0.207 \AA^{-1}$ and the mixing parameter of $\alpha=0.25$.

\section{RESULTS AND DISCUSSION}

Bulk Ge has an indirect band gap with the valence band maximum (VBM) and conduction band minimum (CBM) states located at the $\Gamma_{b}$ and $L_{b}$ points, respectively, as illustrated in Figure 1a (henceforth, the subscript $b$ is referred to as the $k$ points in the bulk BZ). The 4-fold-degenerate CBM states split into a nondegenerate $\varepsilon_{s}\left(L_{b}\right)$ state and 3-fold-degenerate $\varepsilon_{t}\left(L_{b}\right)$ states under uniaxial strain along the $[111]$ direction. As strain increases, $\varepsilon_{s}\left(L_{b}\right)$ moves up, whereas $\varepsilon_{t}\left(L_{b}\right)$ moves down with respect to the VBM. On the other hand, the CBM energy at $\Gamma_{b}$ decreases faster than the $\varepsilon_{t}\left(L_{b}\right)$ states under the strain. In the absence of strain, experimentally, the difference $(\Delta)$ between the conduction band energies at the $\Gamma_{b}$ and $L_{b}$ points is 0.141 $\mathrm{eV}$ at room temperature (RT) and $0.154 \mathrm{eV}$ at $1.5 \mathrm{~K}^{14,23}$ For these measured values for $\Delta$, we performed the HSE06 calculations with including the effects of normal compression and internal strain. We find that the critical strains required for the indirect-to-direct band gap transition are $3.8 \%$ and $4.1 \%$ at
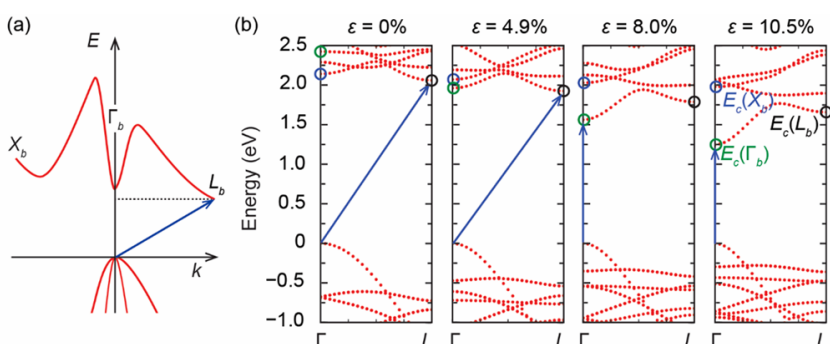

(c)

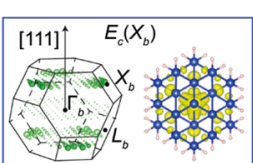

(d)

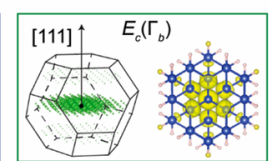

(e)

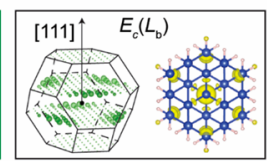

Diameter $(\mathrm{nm})$
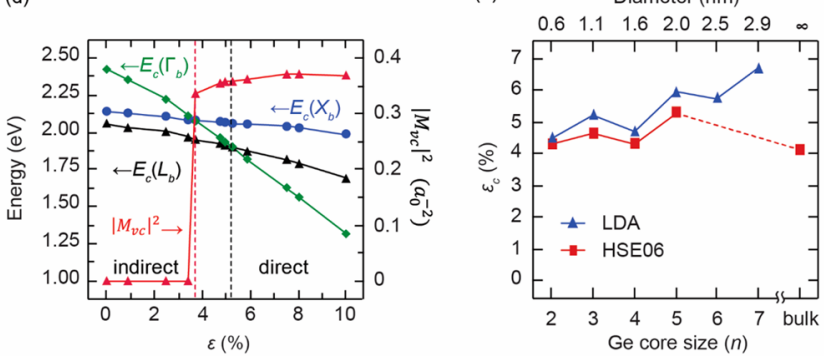

Figure 1. (a) A schematic band diagram of bulk Ge and (b) the LDA band structures of the [111]-oriented $\mathrm{Ge}_{3} \mathrm{NW}$ with the diameter of $10.86 \AA$ under various uniaxial strains $(\varepsilon)$ along the axial direction. Green, blue, and black circles indicate the conduction band states of Ge NW, $E_{c}\left(\Gamma_{b}\right), E_{c}\left(X_{b}\right)$, and $E_{c}\left(L_{b}\right)$, respectively. (c) The charge distributions for the $E_{c}\left(\Gamma_{b}\right), E_{c}\left(X_{b}\right)$, and $E_{c}\left(L_{b}\right)$ states and their orbital characteristics which represent the degree of contributions from the $\Gamma_{b}, X_{b}$, and $L_{b}$ points in the bulk BZ. (d) The variations of the $E_{c}\left(\Gamma_{b}\right)$, $E_{c}\left(L_{b}\right)$, and $E_{c}\left(X_{b}\right)$ states and the square of the dipole matrix element of the direct transition at the $\Gamma$ point under uniaxial strain along the wire axis. Red and black vertical dashed lines represent the strains where the $E_{c}\left(\Gamma_{b}\right)$ state crosses with the $E_{c}\left(X_{b}\right)$ and $E_{c}\left(L_{b}\right)$ states, respectively. (e) The results of the LDA and HSE06 calculations for the critical strain $\left(\varepsilon_{c}\right)$ at which an indirect-to-direct band gap transition occurs in pure Ge NWs consisting of $n$ Ge layers and bulk Ge.

RT and $1.5 \mathrm{~K}$, respectively, in good agreement with the previous $\mathrm{HSE}^{23} 6^{23}$ and quasiparticle $G W$ calculations. $^{20}$

The band structure of [111]-oriented Ge NWs can be understood in terms of the zone folding of the bulk BZ along the [111] direction. The $L_{b}$ point is projected onto the $L$ point on the boundary of the NW BZ, while the $\Gamma_{b}$ and $X_{b}$ points are projected onto the center of the BZ ( $\Gamma$ point). Thus, the CBM and VBM states of [111]-oriented Ge NWs will be located at the $L$ and $\Gamma$ points, respectively. The LDA band structure of Ge NW with the diameter of $10.86 \AA$ is shown in Figure $1 \mathrm{~b}$. The axial lattice constant is slightly shortened, as compared to bulk Ge. Nevertheless, it is clear that Ge NW has an indirect band gap in the absence of strain. By using the band-unfolding method $^{50-52}$ and analyzing the orbital characteristics, we confirm that the VBM and CBM states of Ge NW are derived from the $\Gamma_{b}$ and $L_{b}$ points, respectively. While two lowest conduction band states at the $\Gamma$ point are folded from the $\Gamma_{b}$ and $X_{b}$ points, the conduction band derived from the $\Gamma_{b}$ point is higher than that derived from the $X_{b}$ point, in contrast to bulk Ge where the $X_{b}$ point is higher in energy than the $\Gamma_{b}$ point. Here, we denote $E_{c}\left(\Gamma_{b}\right), E_{c}\left(L_{b}\right)$, and $E_{c}\left(X_{b}\right)$ for the conduction band states of Ge NW, which are derived from the $\Gamma_{b}, L_{b}$, and $X_{b}$ points, respectively (Figure $\left.1 c\right)$. The variations of $E_{c}\left(\Gamma_{b}\right)$, $E_{c}\left(L_{b}\right)$, and $E_{c}\left(X_{b}\right)$ with uniaxial strain are shown in Figure $1 \mathrm{~b}$. 
Table 1. Diameters and Band Gaps of $\mathrm{Ge}_{n} \mathrm{NWs}$ and $\mathrm{Ge}_{n}$ / $\mathrm{Sn}_{m}$ Core-Shell NWs ${ }^{a}$

\begin{tabular}{|c|c|c|c|c|c|c|c|}
\hline \multirow[b]{2}{*}{ nanowire } & \multirow[b]{2}{*}{$d_{\text {core }}$} & \multirow[b]{2}{*}{$d_{\text {total }}$} & \multicolumn{2}{|c|}{ LDA } & \multicolumn{2}{|c|}{ HSE06 } & \multirow[b]{2}{*}{ gap } \\
\hline & & & $E_{\mathrm{D}}(\Gamma)$ & $E_{\mathrm{ID}}(L)$ & $E_{\mathrm{D}}(\Gamma)$ & $E_{\mathrm{ID}}(L)$ & \\
\hline $\mathrm{Ge}_{2}$ & & 6.27 & 3.48 & 3.23 & 4.26 & 4.03 & ID \\
\hline $\mathrm{Ge}_{2} / \mathrm{Sn}_{1}$ & 6.85 & 12.14 & 1.91 & 1.93 & 2.42 & 2.51 & $\mathrm{D}$ \\
\hline $\mathrm{Ge}_{2} / \mathrm{Sn}_{2}$ & 7.01 & 17.52 & 1.01 & 1.24 & 1.36 & 1.68 & $\mathrm{D}$ \\
\hline $\mathrm{Ge}_{2} / \mathrm{Sn}_{3}$ & 6.87 & 22.73 & 0.42 & 0.66 & & & $\mathrm{D}$ \\
\hline $\mathrm{Ge}_{3}$ & & 10.86 & 2.14 & 2.06 & 2.88 & 2.78 & ID \\
\hline $\mathrm{Ge}_{3} / \mathrm{Sn}_{1}$ & 11.52 & 16.74 & 1.37 & 1.34 & 1.88 & 1.87 & ID \\
\hline $\mathrm{Ge}_{3} / \mathrm{Sn}_{2}$ & 11.44 & 22.26 & 0.74 & 0.80 & & & $\mathrm{D}$ \\
\hline $\mathrm{Ge}_{3} / \mathrm{Sn}_{3}$ & 11.85 & 27.74 & 0.25 & 0.37 & & & $\mathrm{D}$ \\
\hline $\mathrm{Ge}_{4}$ & & 15.50 & 1.59 & 1.50 & 2.27 & 2.16 & ID \\
\hline $\mathrm{Ge}_{4} / \mathrm{Sn}_{1}$ & 16.28 & 21.44 & 1.10 & 1.07 & & & ID \\
\hline $\mathrm{Ge}_{4} / \mathrm{Sn}_{2}$ & 16.34 & 27.12 & 0.59 & 0.64 & & & $\mathrm{D}$ \\
\hline $\mathrm{Ge}_{4} / \mathrm{Sn}_{3}$ & 16.38 & 32.48 & 0.10 & 0.22 & & & $\mathrm{D}$ \\
\hline $\mathrm{Ge}_{5}$ & & 20.10 & 1.26 & 1.09 & 1.92 & 1.74 & ID \\
\hline $\mathrm{Ge}_{5} / \mathrm{Sn}_{1}$ & 20.80 & 26.05 & 0.89 & 0.75 & & & ID \\
\hline $\mathrm{Ge}_{5} / \mathrm{Sn}_{2}$ & 20.89 & 31.81 & 0.40 & 0.36 & & & ID \\
\hline $\mathrm{Ge}_{5} / \mathrm{Sn}_{3}$ & 21.09 & 37.51 & 0.05 & 0.06 & & & $\mathrm{D}$ \\
\hline $\mathrm{Ge}_{6}$ & & 24.82 & 1.09 & 0.87 & & & ID \\
\hline $\mathrm{Ge}_{6} / \mathrm{Sn}_{1}$ & 25.36 & 30.81 & 0.76 & 0.64 & & & ID \\
\hline
\end{tabular}

${ }^{a}$ Here, $d_{\text {core }}$ and $d_{\text {total }}$ denote the core and total diameters in units of $\AA$, respectively, and $E_{\mathrm{D}}(\Gamma)$ and $E_{\mathrm{ID}}(L)$ represent the direct $(\mathrm{D})$ and indirect (ID) band gaps in units of $\mathrm{eV}$ at the $\Gamma$ and $L$ points, respectively, which were obtained from the LDA and HSE06 calculations.

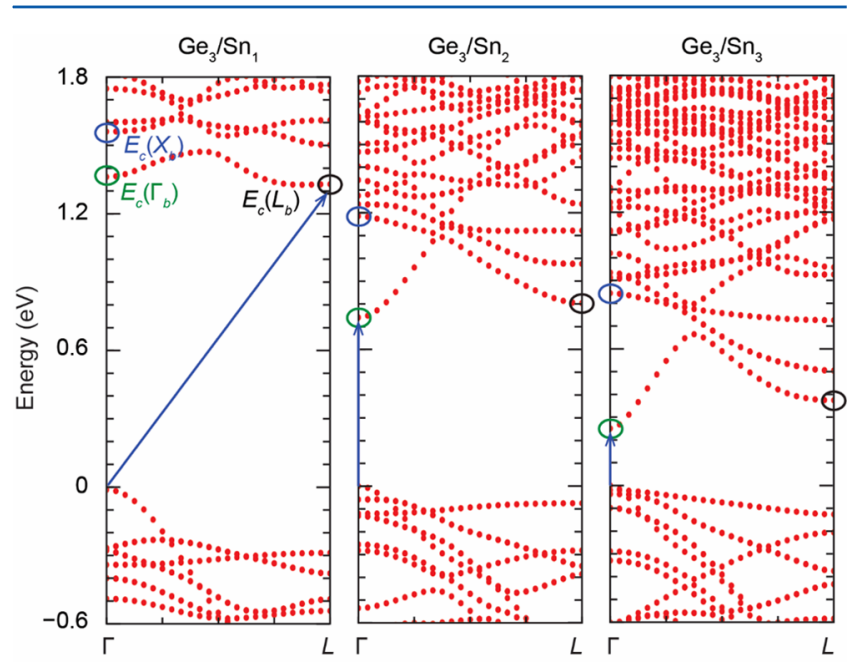

Figure 2. LDA band structures of $\mathrm{Ge}_{3} / \mathrm{Sn}_{1}, \mathrm{Ge}_{3} / \mathrm{Sn}_{2}$, and $\mathrm{Ge}_{3} / \mathrm{Sn}_{3}$ core-shell NWs without external strain. Green, blue, and black circles indicate the conduction band states, $E_{c}\left(\Gamma_{b}\right), E_{c}\left(X_{b}\right)$, and $E_{c}\left(L_{b}\right)$, which have the $\Gamma_{b^{-}}, X_{b}$, and $L_{b}$-like characters of bulk Ge, respectively.

While all the states tend to decrease with increasing of strain, the downward shift of $E_{c}\left(\Gamma_{b}\right)$ is more significant (Figure 1d), similar to bulk Ge. ${ }^{21,22}$ The $E_{c}\left(\Gamma_{b}\right)$ state first crosses with the $E_{c}\left(X_{b}\right)$ state at $3.7 \%$ strain and then with the $E_{c}\left(L_{b}\right)$ state at $5.2 \%$ strain, leading to an indirect-to-direct band gap transition. We examined the optical transition by calculating the square of the dipole matrix element of the direct transition at the $\Gamma$ point, $\left|M_{v c}\right|^{2}=\left|\left\langle\varphi_{c}^{\Gamma}|\hat{p}| \varphi_{v}^{\Gamma}\right\rangle\right|^{2}$. We find that $\left|M_{v c}\right|^{2}$ is rapidly enhanced when the band crossing between the $E_{c}\left(\Gamma_{b}\right)$ and $E_{c}\left(X_{b}\right)$ states occurs (Figure 1d), indicating that the $E_{c}\left(\Gamma_{b}\right)$ state allows for the dipole transition. (a)

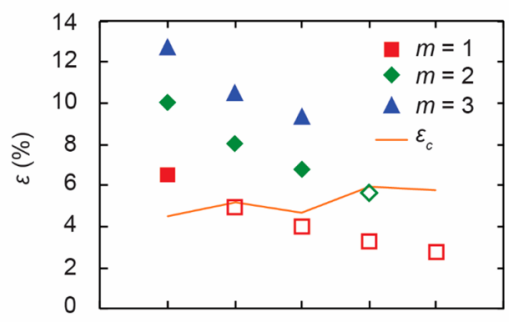

(b)

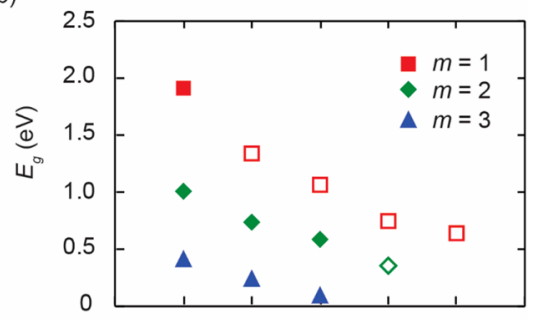

(c)

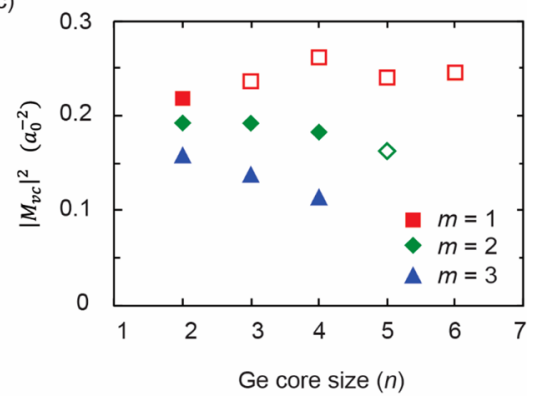

Figure 3. (a) The intrinsic tensile strains $(\varepsilon)$, (b) the LDA band gaps $\left(E_{g}\right)$, and (c) the squares of the dipole matrix elements of the direct transition at the $\Gamma$ point $\left(\left|M_{v c}\right|^{2}\right)$ in [111]-oriented $\mathrm{Ge}_{n} / \mathrm{Sn}_{m}$ core-shell NWs with $n$ Ge layers in the core and $m$ Sn layers in the shell. The orange line in (a) represents the critical strains $\left(\varepsilon_{c}\right)$ for the indirect-todirect transition in pure Ge NWs with the same Ge cores. Filled and unfilled markers stand for the NW structures with the direct and indirect band gaps, respectively.

Figure le shows the critical strain required for the indirectto-direct band gap transition in [111]-oriented Ge NWs with the diameters of about 6.27-29.01 $\AA$. For diameters up to $20.10 \AA$, the critical strain obtained from HSE06 are in the range of $4.3-5.3 \%$, close to the LDA results of $4.5-5.9 \%$. For the Ge NW with the diameter of $10.86 \AA$, the critical strain of about $5.3 \%$ was reported, ${ }^{20}$ in good agreement with our LDA calculations. It is noticeable that the critical strains in Ge NWs are higher than the HSE06 result of $4.1 \%$ for bulk Ge. In the nanowire, the energy difference between the $E_{c}\left(\Gamma_{b}\right)$ and the $E_{c}\left(L_{b}\right)$ states is larger than that of bulk Ge because the $L_{b}$-valley states split by the symmetry breaking. Consequently, a larger tensile strain is required to drive the indirect-to-direct transition.

For bulk Ge, tensile strain along the [100] direction also drives an indirect-to-direct transition. ${ }^{22,23}$ However, for the [100]-oriented Ge NW with the diameter of about $20.81 \AA$, the indirect-to-direct transition does not take place under uniaxial strain up to $10 \%$ (see Figure S1 in the Supporting Information). On the other hand, the [110]-oriented Ge NW with the diameter of about $21.88 \AA$ has a direct band gap even in the absence of strain. However, its optical transition at the threshold energy is not dipole-allowed because the CBM state has the $L_{b}$-like character. Moreover, since the VBM state shifts away from the $\Gamma$ point under uniaxial strain along the $[110]$ 

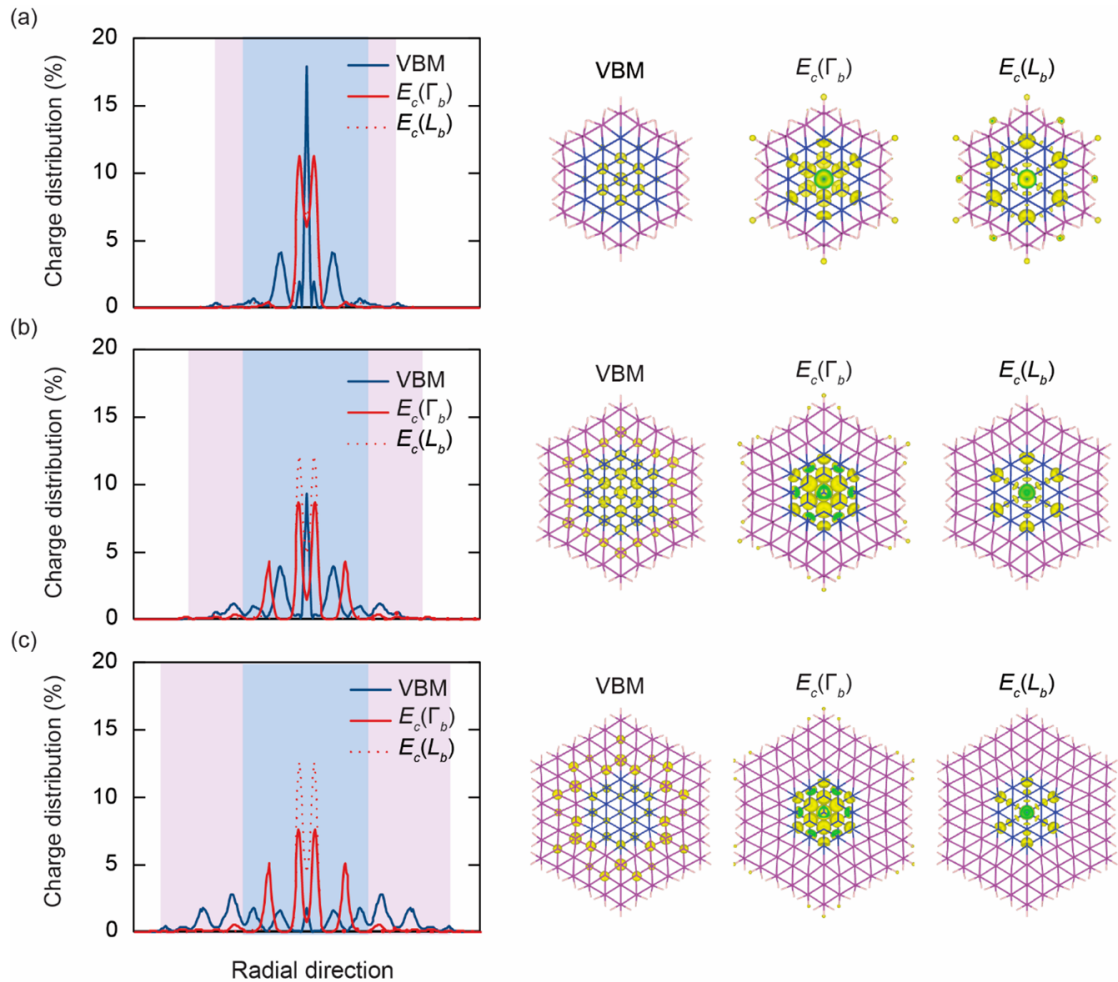

Figure 4. (left) The charge distributions of the valence band maximum $(\mathrm{VBM})$ and the conduction band edge states at the $\Gamma$ and $L$ points, $E_{c}\left(\Gamma_{b}\right)$ and $E_{c}\left(L_{b}\right)$, along the radial direction and (right) their spatial distributions over the wire cross section in (a) $\mathrm{Ge}_{3} / \mathrm{Sn}_{1},(\mathrm{~b}) \mathrm{Ge}_{3} / \mathrm{Sn}_{2}$, and $(\mathrm{c}) \mathrm{Ge}_{3} / \mathrm{Sn}_{3}$ core-shell NWs. Blue, purple, and white backgrounds in the left panel correspond to the Ge core, Sn shell, and vacuum regions, respectively.

direction, the band gap changes from direct to indirect (see Figure S2 in the Supporting Information).

We propose a different route to achieve direct band gap nanowires by constructing $\mathrm{Ge} / \mathrm{Sn}$ core-shell NWs with the radial heterostructure. Since the lattice constant of $\mathrm{Sn}$ is larger than that of Ge, the $\mathrm{Sn}$ shell induces tensile strain on the Ge core. We considered [111]-oriented $\mathrm{Ge}_{n} / \mathrm{Sn}_{m}$ core-shell NWs, where the Ge core of $n$ layers $(n=2-6)$ is wrapped with the $S n$ shell of $m$ layers $(m=1-3)$. The nanowire diameters range from 12.14 to $37.51 \AA$ (Table 1 ). For a given Ge core size, we find a tendency that the indirect band gap turns to the direct band gap as the shell thickness increases. To confirm that, the band structures of $\mathrm{Ge}_{3} / \mathrm{Sn}_{m}$ core-shell NWs are compared with those for tensile-strained $\mathrm{Ge}_{3} \mathrm{NWs}$ with only the Ge core of three layers. While the $\mathrm{Ge}_{3} / \mathrm{Sn}_{1} \mathrm{NW}$ has the indirect band gap of $1.34 \mathrm{eV}$, the direct band gaps of 0.74 and $0.25 \mathrm{eV}$ occur for the $\mathrm{Ge}_{3} / \mathrm{Sn}_{2}$ and $\mathrm{Ge}_{3} / \mathrm{Sn}_{3} \mathrm{NWs}$, respectively (Figure 2). For the $\mathrm{Ge}_{3} / \mathrm{Sn}_{1}, \mathrm{Ge}_{3} / \mathrm{Sn}_{2}$, and $\mathrm{Ge}_{3} / \mathrm{Sn}_{3} \mathrm{NWs}$, the intrinsic strains are estimated to be $4.9,8.0$, and $10.5 \%$, respectively, when the equilibrium lattice constant of the pure $\mathrm{Ge}_{3} \mathrm{NW}$ is used as a reference. The $\mathrm{Ge}_{3} \mathrm{NW}$ exhibits the same tendency that the indirect band gap decreases as strain increases up to $4.9 \%$, and it turns to the direct band gap for higher strains of $8.0 \%$ and $10.5 \%$ (Figure $1 \mathrm{~b}$ ). It is verified that $\mathrm{Ge} / \mathrm{Sn}$ core-shell NWs have direct band gaps when their intrinsic tensile strains are larger than the critical values for Ge NWs with only the Ge cores (Figure 1e and Figure 3a). In addition, we find that the intrinsic tensile strain induced on the Ge core is inversely proportional to the core size, whereas it is proportional to the $\mathrm{Sn}$ shell thickness. For Ge/Sn core-shell NWs with large diameters, which cannot be handled within the first-principles LDA calculations, we estimate the intrinsic strain induced on the Ge core by using a continuum elasticity theory ${ }^{53}$ (see Figure S3 in the Supporting Information). For small-diameter NWs, we find good agreements between the two different calculations.

The band gap of Ge/Sn core-shell NWs can be tuned by controlling the diameter and the core-to-shell ratio, as shown in Figure $3 \mathrm{~b}$. As the $\mathrm{Sn}$ shell becomes thicker, the contribution of the Sn orbitals to the band edge states is enhanced, resulting in the reduction of the band gap because $\mathrm{Sn}$ has zero band gap. ${ }^{54}$ Moreover, since the thicker $S n$ shell increases the intrinsic strain on the Ge core, the band gap is also reduced. On the other hand, for a given shell thickness, the band gap increases as the $\mathrm{Ge}$ core size decreases due to the stronger quantum confinement effect. For $\mathrm{Ge}_{n} / \mathrm{Sn}_{m}$ core-shell NWs with the diameters of $12.14-37.51 \AA(n=2-6$ and $m=1-3)$, all the band structures are shown in Figure $S 4$ in the Supporting Information. The direct band gaps calculated by the LDA range from 0.05 to $1.91 \mathrm{eV}$ (Table 1). For selected NWs with the diameters less than $18 \AA$, the band gaps increase by $27-40 \%$ with the hybrid HSE06 functional, exhibiting the largest band gap of $2.42 \mathrm{eV}$ for $n=2$ and $m=1$. The variable direct band gaps of about $1 \mathrm{eV}$ will be suitable for applications including optical communications. Experiments have reported the synthesis of Ge NWs with the diameters of about $3 \mathrm{~nm}^{55}$ For the pure $\mathrm{Ge}_{7} \mathrm{NW}$ with the diameter of about $3 \mathrm{~nm}$, the indirect-to-direct transition takes place at about $6.3 \%$ strain and the band gap is estimated to be about $1 \mathrm{eV}$ at the critical strain (see Figure S5 in the Supporting Information). According to the continuum elasticity model (see Figure S3 in the Supporting Information), the Sn shell of about $1 \mathrm{~nm}$ thickness $(m \sim 4)$ is required to induce the intrinsic strain of $6.3 \%$ on the $\mathrm{Ge}_{7}$ core. With including a band gap correction for the pure 
(a)
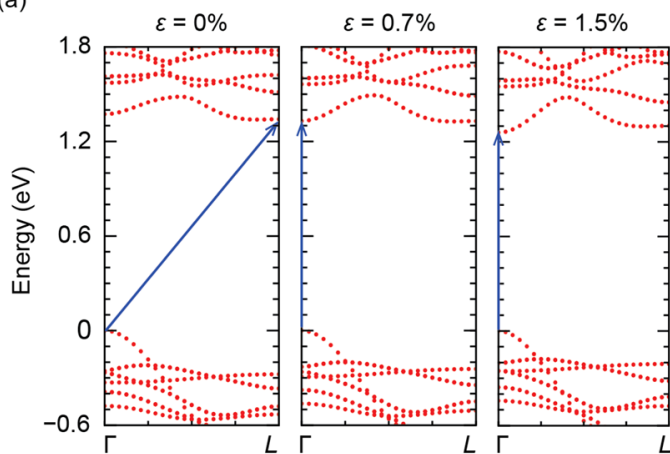

(b)

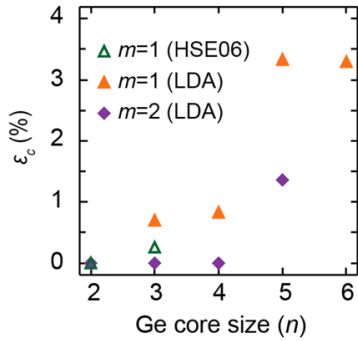

(c)

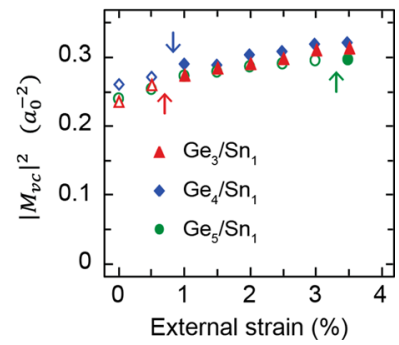

Figure 5. (a) The variation of the LDA band structure of the $\mathrm{Ge}_{3} / \mathrm{Sn}_{1}$ core-shell NW under external tensile strain $(\varepsilon)$. The indirect-to-direct band gap transition occurs at $0.7 \%$ strain. (b) The external critical strains $\left(\varepsilon_{c}\right)$ for the indirect-to-direct band gap transition in $\mathrm{Ge}_{n} / \mathrm{Sn}_{1}$ and $\mathrm{Ge}_{n} / \mathrm{Sn}_{2}$ core-shell NWs from the LDA and HSE06 calculations. External strain is defined with respect to the equilibrium lattice constants of $\mathrm{Ge} / \mathrm{Sn}$ core-shell NWs. (c) Squares of dipole matrix element for the direct transition at the $\Gamma$ point as a function of external strain. Filled and empty symbols in (c) denote the direct and indirect band gaps, respectively, and arrows indicate the critical strains where the indirect-to-direct gap transition occurs.

$\mathrm{Ge}_{7} \mathrm{NW}$, we expect that the band gaps of $\mathrm{Ge}_{7} / \mathrm{Sn}_{m}$ core-shell NWs with $m=0-4$ lie in the range of $0.3-1.3 \mathrm{eV}$ (see Table S2 and Figure S6 in the Supporting Information).

The dipole allowedness of the direct transition at the $\Gamma$ point is examined by calculating the square of the dipole matrix element $\left|M_{v c}\right|^{2}$. For Ge/Sn core-shell NWs with direct band gaps, the squared dipole matrix elements were calculated to be $0.11-0.22 a_{0}^{-2}$ in atomic units (Figure $3 \mathrm{c}$ ), where $a_{0}$ is the Bohr radius. These values are much higher than $0.03 a_{0}^{-2}$ obtained for a specially designed $\mathrm{Si} / \mathrm{Ge}$ superstructure, ${ }^{18}$ while they are slightly lower than that of GaAs $\left(0.31 a_{0}^{-2}\right) .{ }^{56}$ When the core size is fixed, the squared dipole matrix elements tend to decrease as the shell thickness increases. To understand this trend, we examined the charge distributions of the energy states near the band gap for $\mathrm{Ge}_{3} / \mathrm{Sn}_{1}, \mathrm{Ge}_{3} / \mathrm{Sn}_{2}$, and $\mathrm{Ge}_{3} / \mathrm{Sn}_{3} \mathrm{NWs}$ (Figure 4). We find that the $E_{c}\left(\Gamma_{b}\right)$ and $E_{c}\left(L_{b}\right)$ states are mainly distributed over the Ge core, regardless of the shell size. On the other hand, as the Sn shell becomes thicker, the charge distribution of the VBM state extends to the shell region, while the CBM state maintains the localization in the core region. Thus, the overlap between the VBM and CBM states decreases, reducing the optical transition probability. We note that $\mathrm{Ge} / \mathrm{Sn}$ core-shell NWs with thin Sn shells exhibit the band alignment of type-I, although bulk $\mathrm{Sn}$ has the metallic band structure. The type-I band alignment is attributed to the nonzero band gap of the $\mathrm{Sn}$ shell, which results from the strong quantum confinement effect, similar to pure Sn NWs (see Table S2 in the Supporting Information).
We investigate the effect of external tensile strain on the indirect-to-direct transition in Ge/Sn core-shell NWs with indirect band gaps. If the $\mathrm{Sn}$ shell consists of only one layer, $\mathrm{Ge}_{n} / \mathrm{Sn}_{1}$ core-shell NWs have intrinsically indirect band gaps for $n \geq 3$ (Table 1 ). We find that the external critical strain is significantly reduced in $\mathrm{Ge} / \mathrm{Sn}$ core-shell NWs, compared with pure Ge NWs. For example, the LDA critical strain is estimated to be $0.7 \%$ for the $\mathrm{Ge}_{3} / \mathrm{Sn}_{1}$ core-shell NW (Figure 5a,b), while it is about $5.2 \%$ in the pure $\mathrm{Ge}_{3} \mathrm{NW}$ with the same core size (Figure 1e). Moreover, external strain also slightly improves the optical transition in Ge/Sn core-shell NWs, as shown in Figure 5c. When $0.7 \%$ tensile strain is applied to the $\mathrm{Ge}_{3} / \mathrm{Sn}_{1}$ coreshell $\mathrm{NW}$, the square of the dipole matrix element for the direct transition at the $\Gamma$ point increases from 0.24 to $0.27 a_{0}^{-2}$. Similar strain effects are found for $\mathrm{Ge}_{n} / \mathrm{Sn}_{1}$ core-shell NWs with $n \geq$ 4. However, the critical strain tends to increase as the core size increases, as shown in Figure 5b, while its values are generally lower than those for pure Ge NWs and bulk Ge.

Finally, we discuss the possible synthesis of $\mathrm{Ge} / \mathrm{Sn}$ coreshell NWs. We compare the formation energy of the $\mathrm{Ge}_{4} / \mathrm{Sn}_{2}$ core-shell NW with those of GeSn alloy NWs with the same Sn content. We generate five GeSn alloy NWs, in which the Ge and $\mathrm{Sn}$ atoms are randomly distributed, whereas the outermost shells are composed of the $\mathrm{Sn}$ atoms to avoid the formation of different $\mathrm{Ge}-\mathrm{H}$ and $\mathrm{Sn}-\mathrm{H}$ bonds on the wire surface (see Figure S7 in the Supporting Information). We find that the average energy of the GeSn alloy NWs is lower by about 40 $\mathrm{meV}$ per $\mathrm{Sn}$ atom than that of the core-shell NW. On the other hand, an isolated $\mathrm{Sn}$ atom is energetically more favorable near the surface in pure Ge NWs due to the local strain effect. Moreover, the activation enthalpy for the diffusion of $\mathrm{Sn}$ in $\mathrm{Ge}$ is around $3 \mathrm{eV} .{ }^{57}$ Considering the small energy difference between the core-shell and alloy NWs, we do not rule out the possibility that the $\mathrm{Ge} / \mathrm{Sn}$ core-shell structure can be synthesized by a controlled epitaxial growth of the Sn shell on the Ge NW, similar to other core-shell or multishell nanowires. ${ }^{58}$ Our results are also supported by recent experiments reported that uniaxial tensile strain can be achieved in $\mathrm{Ge} / \mathrm{GeSn}$ multishells grown on a one-dimensional Si pillar or cavity, leading to the indirect-to-direct gap transition of $\mathrm{Ge} .^{59}$

\section{CONCLUSIONS}

In conclusion, we have shown that the dipole-allowed direct band gaps can be achieved by wrapping [111]-oriented $\mathrm{Ge}$ NWs with Sn layers in the form of core-shell NWs with the radial heterostructure, without applying an external strain. The band gaps can be tuned by controlling the diameter and the core-to-shell ratio. The indirect-to-direct band gap transition is attributed to the intrinsic tensile strain induced by the lattice mismatch between the Ge core and the $\mathrm{Sn}$ shell. Due to the band crossing between the bulk $\Gamma_{b^{-}}$and $L_{b}$-derived states, the optical transitions of the direct band gaps are all dipole-allowed. For Ge/Sn core-shell NWs with intrinsically indirect band gaps, an external strain can be used to induce the indirect-todirect transition; however, the critical values are much lower than those for bulk Ge and pure Ge NWs with the same core sizes. The dipole allowedness of the direct band gaps suggests that [111]-oriented $\mathrm{Ge} / \mathrm{Sn}$ core-shell NWs can serve as potential candidate materials for light emitters. 


\section{ASSOCIATED CONTENT}

\section{S Supporting Information}

The Supporting Information is available free of charge on the ACS Publications website at DOI: 10.1021/acs.jpcc.6b08779.

The band structures of [100]- and [110]-oriented Ge NWs under uniaxial tensile strain, the detailed modeling of intrinsic strain on large-sized Ge/Sn core-shell NWs, based on the elasticity theory, the band structures of $\mathrm{Ge}$ / Sn core-shell NWs with different core sizes and core-toshell ratios, the variation of the band gap of $\mathrm{Ge}_{7} \mathrm{NW}$ with the diameter of $\sim 3 \mathrm{~nm}$ under strain, the expected band gaps with gap corrections of Ge/Sn core-shell NWs, and the atomic structures of GeSn alloy NWs (PDF)

\section{AUTHOR INFORMATION}

\section{Corresponding Author}

*E-mail: kjchang@kaist.ac.kr. Phone: 82-42-350-2531. Fax: 8242-350-2510.

\section{ORCID ${ }^{\circ}$}

K. J. Chang: 0000-0002-5364-8551

\section{Author Contributions}

E.P., S.K., and K.J.C. contributed equally to this manuscript. K.J.C. conceived the work and designed the research strategy. E.P. performed theoretical calculations, and E.P. and S.K. did data analysis. All authors discussed the results and co-wrote the manuscript.

Notes

The authors declare no competing financial interest.

\section{ACKNOWLEDGMENTS}

This work was supported by Samsung Science and Technology Foundation under Grant No. SSTF-BA1401-08.

\section{REFERENCES}

(1) Ma, D. D. D.; Lee, C. S.; Au, F. C. K.; Tong, S. Y.; Lee, S. T. Small-Diameter Silicon Nanowire Surfaces. Science 2003, 299, 18741877.

(2) Zhong, Z.; Wang, D.; Cui, Y.; Bockrath, M. W.; Lieber, C. M. Nanowire Crossbar Arrays as Address Decoders for Integrated Nanosystems. Science 2003, 302, 1377-1379.

(3) Pillarisetty, R. Academic and Industry Research Progress in Germanium Nanodevices. Nature 2011, 479, 324-328.

(4) Niquet, Y.-M.; Delerue, C. Carrier Mobility in Strained Ge Nanowires. J. Appl. Phys. 2012, 112, 084301.

(5) Wang, D.; Wang, Q.; Javey, A.; Tu, R; Dai, H.; Kim, H.; McIntyre, P. C.; Krishnamohan, T.; Saraswat, K. C. Germanium Nanowire Field-Effect Transistors with $\mathrm{SiO}_{2}$ and High- $\kappa \mathrm{HfO}_{2}$ Gate Dielectrics. Appl. Phys. Lett. 2003, 83, 2432-2434.

(6) Greytak, A. B.; Lauhon, L. J.; Gudiksen, M. S.; Lieber, C. M. Growth and Transport Properties of Complementary Germanium Nanowire Field-Effect Transistors. Appl. Phys. Lett. 2004, 84, 41764178.

(7) Ahn, Y. H.; Park, J. Efficient Visible Light Detection Using Individual Germanium Nanowire Field Effect Transistors. Appl. Phys. Lett. 2007, 91, 162102.

(8) Polyakov, B.; Daly, B.; Prikulis, J.; Lisauskas, V.; Vengalis, B.; Morris, M. A.; Holmes, J. D.; Erts, D. High-Density Arrays of Germanium Nanowire Photoresistors. Adv. Mater. 2006, 18, 18121816.

(9) Chan, C. K.; Zhang, X. F.; Cui, Y. High Capacity Li Ion Battery Anodes Using Ge Nanowires. Nano Lett. 2008, 8, 307-309.

(10) Zahedifar, M.; Hosseinmardi, F.; Eshraghi, L.; Ganjipour, B. Synthesis and Thermoluminescence of Boron-Doped Germanium Nanowires. Radiat. Phys. Chem. 2011, 80, 324-327.
(11) Patibandla, S.; Pramanik, S.; Bandyopadhyay, S.; Tepper, G. C. Spin Relaxation in a Germanium Nanowire. J. Appl. Phys. 2006, 100, 044303.

(12) Ye, H.; Yu, J. Germanium Epitaxy on Silicon. Sci. Technol. Adv. Mater. 2014, 15, 024601.

(13) Zhou, Z.; Yin, B.; Michel, J. On-Chip Light Sources for Silicon Photonics. Light: Sci. Appl. 2015, 4, e358.

(14) Geiger, R.; Zabel, T.; Sigg, H. Group IV Direct Band Gap Photonics: Methods, Challenges, and Opportunities. Front. Mater. 2015, 2, 52

(15) Medaboina, D.; Gade, V.; Patil, S. K. R.; Khare, S. V. Effect of Structure, Surface Passivation, and Doping on the Electronic Properties of Ge Nanowires: A First-Principles Study. Phys. Rev. B: Condens. Matter Mater. Phys. 2007, 76, 205327.

(16) Lee, A. J.; Kim, M.; Lena, C.; Chelikowsky, J. R. Mechanical and Electronic Properties of Strained Ge Nanowires Using Ab Initio RealSpace Pseudopotentials. Phys. Rev. B: Condens. Matter Mater. Phys. 2012, 86, 115331.

(17) Zhang, L.; d'Avezac, M.; Luo, J.-W.; Zunger, A. Genomic Design of Strong Direct-Gap Optical Transition in Si/Ge Core/Multishell Nanowires. Nano Lett. 2012, 12, 984-991.

(18) d'Avezac, M.; Luo, J.-W.; Chanier, T.; Zunger, A. GeneticAlgorithm Discovery of a Direct-Gap and Optically Allowed Superstructure from Indirect-Gap Si and Ge Semiconductors. Phys. Rev. Lett. 2012, 108, 027401.

(19) Yang, C. H.; Yu, Z. Y.; Liu, Y. M.; Lu, P. F.; Gao, T.; Li, M.; Manzoor, S. Dependence of Electronic Properties of Germanium on the In-Plane Biaxial Tensile Strains. Phys. B 2013, 427, 62-67.

(20) Zhang, F.; Crespi, V. H.; Zhang, P. Prediction that Uniaxial Tension along < 111> Produces a Direct Band Gap in Germanium. Phys. Rev. Lett. 2009, 102, 156401.

(21) Hoshina, Y.; Iwasaki, K.; Yamada, A.; Konagai, M. FirstPrinciples Analysis of Indirect-to-Direct Band Gap Transition of Ge under Tensile Strain. Jpn. J. Appl. Phys. 2009, 48, 04C125.

(22) Liu, L.; Zhang, M.; Hu, L.; Di, Z.; Zhao, S.-J. Effect of Tensile Strain on the Electronic Structure of Ge: A First-Principles Calculation. J. Appl. Phys. 2014, 116, 113105.

(23) Inaoka, T.; Furukawa, T.; Toma, R.; Yanagisawa, S. TensileStrain Effect of Inducing the Indirect-to-Direct Band-Gap Transition and Reducing the Band-Gap Energy of Ge. J. Appl. Phys. 2015, 118, 105704.

(24) Pandey, R.; Rérat, M.; Causà, M. First-Principles Study of Stability, Band Structure, and Optical Properties of the Ordered $\mathrm{Ge}_{0.50}$ $\mathrm{Sn}_{0.50}$ Alloy. Appl. Phys. Lett. 1999, 75, 4127-4129.

(25) Zhang, P.; Crespi, V. H.; Chang, E.; Louie, S. G.; Cohen, M. L. Computational Design of Direct Bandgap Semiconductors that Lattice-Match Silicon. Nature 2001, 409, 69-71.

(26) Low, K. L.; Yang, Y.; Han, G.; Fan, W.; Yeo, Y.-C. Electronic Band Structure and Effective Mass Parameters of $\mathrm{Ge}_{1-\mathrm{x}} \mathrm{Sn}_{\mathrm{x}}$ Alloys. J. Appl. Phys. 2012, 112, 103715.

(27) Lee, M.-H.; Liu, P.-L.; Hong, Y.-A.; Chou, Y.-T.; Hong, J.-Y.; Siao, Y.-J. Electronic Band Structures of $\mathrm{Ge}_{1-\mathrm{x}} \mathrm{Sn}_{\mathrm{x}}$ Semiconductors: A First-Principles Density Functional Theory Study. J. Appl. Phys. 2013, $113,063517$.

(28) von den Driesch, N.; Stange, D.; Wirths, S.; Mussler, G.; Holländer, B.; Ikonic, Z.; Hartmann, J. M.; Stoica, T.; Mantl, S.; Grützmacher, D.; et al. Direct Bandgap Group IV Epitaxy on Si for Laser Applications. Chem. Mater. 2015, 27, 4693-4702.

(29) Wirths, S.; Geiger, R.; von den Driesch, N.; Mussler, G.; Stoica, T.; Mantl, S.; Ikonic, Z.; Luysberg, M.; Chiussi, S.; Hartmann, J. M.; et al. Lasing in Direct-Bandgap GeSn Alloy Grown on Si. Nat. Photonics 2015, 9, 88-92.

(30) Sánchez-Pérez, J. R.; Boztug, C.; Chen, F.; Sudradjat, F. F.; Paskiewicz, D. M.; Jacobson, R.; Lagally, M. G.; Paiella, R. DirectBandgap Light-Emitting Germanium in Tensilely Strained Nanomembranes. Proc. Natl. Acad. Sci. U. S. A. 2011, 108, 18893-18898.

(31) Huo, Y.; Lin, H.; Chen, R.; Makarova, M.; Rong, Y.; Li, M.; Kamins, T. I.; Vuckovic, J.; Harris, J. S. Strong Enhancement of Direct 
Transition Photoluminescence with Highly Tensile-Strained Ge Grown by Molecular Beam Epitaxy. Appl. Phys. Lett. 2011, 98, 011111. (32) Jain, J. R.; Hryciw, A.; Baer, T. M.; Miller, D. A. B.; Brongersma, M. L.; Howe, R. T. A Micromachining-Based Technology for Enhancing Germanium Light Emission via Tensile Strain. Nat. Photonics 2012, 6, 398-405.

(33) Nam, D.; Sukhdeo, D.; Cheng, S.-L.; Roy, A.; Huang, K. C.-Y; Brongersma, M.; Nishi, Y.; Saraswat, K. Electroluminescence from Strained Germanium Membranes and Implications for an Efficient SiCompatible Laser. Appl. Phys. Lett. 2012, 100, 131112.

(34) Capellini, G.; Kozlowski, G.; Yamamoto, Y.; Lisker, M.; Wenger, C.; Niu, G.; Zaumseil, P.; Tillack, B.; Ghrib, A.; de Kersauson, M.; et al. Strain Analysis in SiN/Ge Microstructures Obtained via SiComplementary Metal Oxide Semiconductor Compatible Approach. J. Appl. Phys. 2013, 113, 013513.

(35) Ghrib, A.; El Kurdi, M.; de Kersauson, M.; Prost, M.; Sauvage, S.; Checoury, X.; Beaudoin, G.; Sagnes, I.; Boucaud, P. TensileStrained Germanium Microdisks. Appl. Phys. Lett. 2013, 102, 221112.

(36) El Kurdi, M.; Prost, M.; Ghrib, A.; Sauvage, S.; Checoury, X.; Beaudoin, G.; Sagnes, I.; Picardi, G.; Ossikovski, R.; Boucaud, P. Direct Band Gap Germanium Microdisks Obtained with Silicon Nitride Stressor Layers. ACS Photonics 2016, 3, 443-448.

(37) Sukhdeo, D. S.; Nam, D.; Kang, J.-H.; Brongersma, M. L.; Saraswat, K. C. Direct Bandgap Germanium-on-Silicon Inferred from $5.7 \%<100>$ Uniaxial Tensile Strain. Photonics Res. 2014, 2, A8-A13.

(38) Guilloy, K.; Pauc, N.; Gassenq, A.; Gentile, P.; Tardif, S.; Rieutord, F.; Calvo, V. Tensile Strained Germanium Nanowires Measured by Photocurrent Spectroscopy and X-ray Microdiffraction. Nano Lett. 2015, 15, 2429-2433.

(39) Greil, J.; Lugstein, A.; Zeiner, C.; Strasser, G.; Bertagnolli, E. Tuning the Electro-optical Properties of Germanium Nanowires by Tensile Strain. Nano Lett. 2012, 12, 6230-6234.

(40) Haas, P.; Tran, F.; Blaha, P. Calculation of the Lattice Constant of Solids with Semilocal Functionals. Phys. Rev. B: Condens. Matter Mater. Phys. 2009, 79, 085104.

(41) Ceperley, D. M.; Alder, B. J. Ground State of the Electron Gas by a Stochastic Method. Phys. Rev. Lett. 1980, 45, 566-569.

(42) Perdew, J. P.; Zunger, A. Self-Interaction Correction to DensityFunctional Approximations for Many-Electrons Systems. Phys. Rev. B: Condens. Matter Mater. Phys. 1981, 23, 5048-5079.

(43) Blöchl, P. E. Projected Augmented-Wave Method. Phys. Rev. B: Condens. Matter Mater. Phys. 1994, 50, 17953-17979.

(44) Kresse, G.; Joubert, D. From Ultrasoft Pseudopotentials to the Projector Augmented-Wave Method. Phys. Rev. B: Condens. Matter Mater. Phys. 1999, 59, 1758-1775.

(45) Kresse, G.; Furthmüller, J. Efficient Iterative Schemes for $A b$ Initio Total-Energy Calculations Using a Plane-Wave Basis Set. Phys. Rev. B: Condens. Matter Mater. Phys. 1996, 54, 11169-11186.

(46) Kresse, G.; Furthmüller, J. Efficiency of Ab-Initio Total Energy Calculations for Metals and Semiconductors Using a Plane-Wave Basis Set. Comput. Mater. Sci. 1996, 6, 15-50.

(47) Monkhorst, H. J.; Pack, J. D. Special Points for Brillouin-Zone Integration. Phys. Rev. B 1976, 13, 5188-5192.

(48) Heyd, J.; Scuseria, G. E.; Ernzerhof, M. Hybrid Functionals Based on a Screened Coulomb Potential. J. Chem. Phys. 2003, 118, 8207-8215.

(49) Heyd, J.; Scuseria, G. E.; Ernzerhof, M. Erratum: "Hybrid Functionals Based on a Screened Coulomb Potential" [J. Chem. Phys. 118, 8207 (2003)]. J. Chem. Phys. 2006, 124, 219906.

(50) Popescu, V.; Zunger, A. Extracting $E$ versus $k$ Effective Band Structure from Supercell Calculations on Alloys and Impurities. Phys. Rev. B: Condens. Matter Mater. Phys. 2012, 85, 085201.

(51) Allen, P. B.; Berlijn, T.; Casavant, D. A.; Soler, J. M. Recovering Hidden Bloch Character: Unfolding Electrons, Phonons, and Slabs. Phys. Rev. B: Condens. Matter Mater. Phys. 2013, 87, 085322.

(52) Tomić, M.; Jeschke, H. O.; Valentí, R. Unfolding of Electronic Structure through Induced Representations of Space Groups: Application to Fe-Based Superconductors. Phys. Rev. B: Condens. Matter Mater. Phys. 2014, 90, 195121.
(53) Raychaudhuri, S.; Yu, E. T. Calculation of Critical Dimensions for Wurtzite and Cubic Zinc Blende Coaxial Nanowire Heterostructures. J. Vac. Sci. Technol. B 2006, 24, 2053-2059.

(54) Ansari, L.; Fagas, G.; Colinge, J.-P.; Greer, J. C. A Proposed Confinement Modulated Gap Nanowire Transistor Based on a Metal (Tin). Nano Lett. 2012, 12, 2222-2227.

(55) Simanullang, M.; Usami, K.; Kodera, T.; Uchida, K.; Oda, S. Germanium Nanowires with 3-nm-Diameter Prepared by Low Temperature Vapour-Liquid-Solid Chemical Vapour Deposition. J. Nanosci. Nanotechnol. 2011, 11, 8163-8168.

(56) Ossicini, S.; Pavesi, L.; Priolo, F. Light Emitting Silicon for Microphotonics; Springer: Berlin, 2003.

(57) Chroneos, A.; Bracht, H.; Grimes, R. W.; Uberuaga, B. P. Vacancy-Mediated Dopant Diffusion Activation Enthalpies for Germanium. Appl. Phys. Lett. 2008, 92, 172103.

(58) Lauhon, L. J.; Gudiksen, M. S.; Wang, D.; Lieber, C. M. Epitaxial Core-Shell and Core-Multishell Nanowire Heterostructures. Nature 2002, 420, 57-61.

(59) Huang, Z.-M.; Huang, W.-Q.; Liu, S.-R.; Dong, T.-G.; Wang, G.; Wu, X.-K.; Qin, C.-J. Emission of Direct-Gap Band in Germanium with Ge-GeSn Layers on One-Dimensional Structure. Sci. Rep. 2016, 6, 24802 . 